\title{
Pelo buraco da fechadura - estudo etnográfico de um grupo de mentoria na escola médica
}

\author{
Through the keyhole - an ethnographic study of a medical school mentoring group \\ Fabiana Verardino Spina' (1) fabianavspina@uol.com.br \\ Patrícia Lacerda Bellodi' (D)|ptbellodi@uol.com.br
}

\begin{abstract}
RESUMO
Introdução: Programas de mentoria vêm sendo reconhecidos por seus benefícios e estão sendo implementados em muitas escolas médicas. No entanto, poucos estudos têm enfocado a compreensão da relação de mentoria em si. Como a relação de mentoria se desenvolve, em tempo real,
\end{abstract} no cotidiano de uma escola médica?

Objetivo: Neste estudo, buscamos observar as interações entre uma mentora e seu grupo de alunos de diferentes anos em seu ambiente natural.

Método: Utilizando uma metodologia qualitativa etnográfica, um grupo de mentoria foi acompanhado por meio de observação participante durante um ano. Ao final do período de observação, realizou-se um grupo focal para validar as notas de campo. A análise temática de conteúdo orientou nossa organização e interpretação dos dados.

Resultado: Não é simples para um grupo de mentoria, composto por alunos de diferentes anos de graduação, reunir-se regularmente e com tempo suficiente para a atividade. Nesse contexto, o mentor é sempre desafiado a estabelecer conexões entre diferentes temas e experiências para dar sentido à experiência do grupo como um todo. Por sua vez, a diversidade do grupo permite discussões estimulantes e ricas sobre o curso, a vida pessoal dos participantes e o futuro profissional. Os alunos veteranos têm um papel fundamental na dinâmica do grupo ao compartilharem suas vivências e motivarem os alunos iniciantes a seguir em frente.

Conclusão: Manter um grupo de mentoria em uma escola médica é uma tarefa complexa e desafiadora. Mentores precisam ser motivados, ter certas características pessoais e receber apoio para essa função. Grupos heterogêneos com alunos de diferentes anos potencializam o desenvolvimento e o suporte entre os integrantes, aliviando as angústias da formação médica. No entanto, a estrutura e a dinâmica do curso de Medicina dificultam muitas vezes o envolvimento dos alunos, impedindo o acesso deles aos efeitos positivos da mentoria.

Palavras-chave: Tutoria; Estudantes de Medicina; Pesquisa Qualitativa; Etnografia.

\begin{abstract}
Introduction: Mentoring programs have been recognized for their benefits and are being implemented in many medical schools. However, few studies have focused on understanding the mentoring relationship itself. How do mentoring relationships develop in real-time in a medical school? Objective: The purpose of this study was to observe the interactions between a mentor and her students from different academic years in their natural environment.

Method: Using a qualitative ethnographic methodology, a mentoring group's meetings were followed through participant observation for a year. At the end of the observation period, a focus group was carried out to validate field notes. Thematic analysis guided the data organization and interpretation.

Results: It was not easy for a mentoring group with students from different undergraduate years to meet regularly and with enough time. The mentor was always challenged to establish connections through different themes and experiences to make sense of the whole group's experience. On the other hand, the group's diversity allowed for exciting and rich discussions about the course, the participants' personal lives, and future. Senior students played an essential role in the group dynamics, sharing their experiences and motivating early-year students.

Conclusions: Conducting a mentoring group in a medical school is challenging task. Mentors need to be motivated, have certain personal characteristics, and receive support for this role. Heterogeneous groups with students from different academic years enhance the exchange of experiences and support among the members, relieving anxieties in their medical training. However, the medical course structure and dynamics harm group functioning, hinder student involvement and, therefore, access to the positive effects of mentoring.
\end{abstract}

Keywords: Mentoring; Medical Students; Qualitative Research; Ethnography.

${ }^{1}$ Universidade de São Paulo, São Paulo, São Paulo, Brasil.

Editora: Lia Marcia Cruz da Silveira

Recebido em 01/03/21; Aceito em 07/04/21.

Avaliado pelo processo de double blind review. 


\section{INTRODUÇÃO}

Programas de mentoria têm sido bastante valorizados por seus efeitos e vêm conquistando espaço em escolas médicas que investem no desenvolvimento integral de seus alunos ${ }^{1}$, apoiando tanto suas necessidades acadêmicas quanto pessoais. Por meio de mentoria, os alunos aprendem com os mais experientes e recebem apoio ao longo da jornada acadêmica. $O$ contato com a nova geração, por sua vez, motiva e rejuvenesce os mentores em sua prática profissional ${ }^{2,3}$.

Embora seja reconhecida como uma via de mão dupla no desenvolvimento pessoal e profissional de mentores e mentorados, a relação de mentoria é complexa e não acontece sem problemas ou dificuldades, envolvendo fatores pessoais e organizacionais ${ }^{4}$. Falta de tempo para reuniões de mentoria e treinamento insuficiente de mentores são desafios enfrentados por diferentes programas de mentoria ${ }^{5}$ quando estes se tornam realidade.

Diretrizes e recomendações ${ }^{6}$ são elaboradas para ajudar os mentores em sua tarefa, mas raramente estão baseadas em pesquisas de alta qualidade. Os estudos na área são, em sua maioria, retrospectivos e as avaliações dos programas frequentemente se baseiam em depoimentos e opiniões ${ }^{7,8}$. Pesquisas em mentoria concentram-se na descrição dos objetivos e da dinâmica de diferentes iniciativas ${ }^{9-12}$ e buscam avaliar o impacto nos alunos ${ }^{13,14}$, nos mentores ${ }^{15}$ e nas escolas ${ }^{16}$. Poucos estudos foram dedicados a compreender a relação de mentoria em $\mathrm{si}^{17-20} \mathrm{e}$, raramente, o processo de mentoria durante o desenvolvimento da relação ${ }^{21,22}$.

O que acontece quando acontece a mentoria? Como se desenvolvem as relações de mentoria no dia a dia de uma escola médica? O que veríamos se pudéssemos "olhar pelo buraco da fechadura"?

Neste estudo, e com esse olhar, acompanhamos o cotidiano dos encontros de uma mentora e seu grupo de alunos, observando suas interações de perto e de dentro. Ao adotarmos essa perspectiva, buscamos aprofundar a compreensão das relações de mentoria desenvolvidas, em especial, no contexto da formação médica.

\section{METODOLOGIA}

Nosso estudo foi desenvolvido com um dos grupos de mentoria realizados na Faculdade de Medicina da Universidade de São Paulo (FMUSP).

Iniciada em 2001, a Mentoria FMUSP23 busca apoiar a jornada do "vir-a-ser médico" de seus alunos promovendo um vínculo mais intenso entre professores e alunos (suporte pessoal) e estimulando a integração de experiências entre alunos dos diversos anos (desenvolvimento profissional). $\mathrm{Na}$ FMUSP, os grupos de mentoria são heterogêneos quanto ao ano acadêmico, e a participação dos alunos é voluntária. São previstos de oito a dez encontros no ano. Os mentores são selecionados entre os professores da faculdade e os médicos do hospital-escola, participam de um treinamento inicial e recebem supervisão ao longo do processo.

Para a exploração de um fenômeno complexo, como a relação de mentoria em grupo, adotamos uma abordagem qualitativa, buscando acessar o campo das relações humanas por meio da compreensão de seus significados.

A etnografia, em especial, foi escolhida por sua proposta de investigação da vida social cotidiana in loco e pelo seu valor em explorar e dar sentido aos "aspectos invisíveis das normas e práticas culturais"24.

Trabalhamos com a metodologia de estudo de caso $^{25}$, pois ela, ao investigar em profundidade um caso concreto, permite compreender tanto situações problemáticas, para análise dos obstáculos, quanto situações bem-sucedidas, para identificação de boas práticas. Trabalhamos com um "grupo típico" considerando os seguintes aspectos: adesão dos alunos na média histórica da atividade (ao redor de 30\%) e mentor com dois anos de experiência no programa, pelo menos. Dentro desse critério, um grupo foi sorteado e concordou em participar voluntariamente da pesquisa.

Por meio da técnica de observação participante ${ }^{26}$, com coleta de dados em ambiente natural e de forma longitudinal, as atividades do grupo de mentoria foram acompanhadas em seu contexto natural. Uma das pesquisadoras acompanhou o grupo de mentoria durante seus encontros ao longo de um ano, adotando a posição de participante-como-observador. Nessa situação, o pesquisador deixa claro aos participantes que sua imersão no campo, compartilhando da vida cotidiana do grupo, tem como limite o tempo da investigação.

A pesquisadora-observadora não era conhecida pelos participantes e foi apresentada a eles pela mentora, depois que a proposta da investigação foi por ela discutida com o grupo de alunos. Buscava interferir o mínimo possível na dinâmica do encontro, tomando poucas e discretas notas e falando apenas quando solicitada.

O roteiro de observação era composto por itens relativos a quem (participantes), onde e quando (espaço e tempo do encontro), como (dinâmicas de interação) e o que (temas discutidos). As observações ocorreram no período de abril de 2009 a março de 2010, num total de dez encontros. As notas (diário de campo) foram tomadas imediatamente após as observações, com a posterior elaboração da narrativa dos encontros.

Para assegurar a qualidade de nossa investigação, ao final do período de observação, foi realizado um grupo focal com os participantes para complementar e validar os registros realizados ${ }^{27}$. $\mathrm{Na}$ análise dos dados, adotando uma perspectiva 
fenomenológica ${ }^{28}$, buscamos descrever os encontros de mentoria tal como experienciados, no sentido de "ir às coisas mesmas", isto é, sem pressupostos teóricos prévios. As categorias empíricas, que emergiram do material, foram estabelecidas como temas e subtemas ${ }^{29}$. Realizamos leituras em paralelo do material, e a construção final das categorias foi definida por consenso.

O estudo foi aprovado pelo Comitê de Ética da FMUSP (Protocolo de Pesquisa no 370/2010), com o consentimento informado dos participantes do grupo de mentoria. Nas transcrições das notas de campo, os participantes são apresentados por meio de letras iniciais maiúsculas, sem qualquer correspondência com seus nomes de origem.

\section{RESULTADOS E DISCUSSÃO}

Organizamos nossos dados buscando descrever os encontros do grupo de mentoria de maneira mais próxima possível de sua dinâmica natural. Para ilustrar os relatos e "dar vida" a eles, apresentaremos citações ilustrativas derivadas das observações e do grupo focal.

\section{Quem se encontrava}

O grupo de mentoria era composto por uma mentora, médica de especialidade clínica (Dra. A), e dez alunos de Medicina de diferentes anos: quatro calouros (alunos B, C, D e E), duas alunas do terceiro ano (R e $S$ ), um aluno do quarto ano (G) e três internos ( $X$ e Y, quinto ano, e $Z$, sexto ano). A participação dos alunos era flutuante ao longo dos encontros.

\section{Onde e quando acontecia}

\section{Um laboratório frio, um encontro quente}

O grupo de mentoria se encontrava em um laboratório de pesquisa, localizado na própria faculdade. O espaço físico era pequeno, estreito, com uma iluminação fria e branca. Dra. A preocupava-se em preparar o lugar, mostrando que esperava seus alunos. Apesar de não constituir uma condição ideal, com a condução firme da mentora e a participação dos alunos, o espaço frio do laboratório transformava-se num espaço quente, de relações vivas, entre pessoas.

\section{Os alunos chegam aos poucos}

A chegada dos alunos à reunião era heterogênea. Os calouros chegavam, na maioria das vezes, nos primeiros quinze minutos da reunião. Os alunos veteranos chegavam mais tarde, de forma gradual, e, às vezes, saíam um pouco mais cedo. Dra. A aguardava a chegada dos alunos com certa expectativa ansiosa: "E esse pessoal que não chega! Será que eles não vêm?!". Também fazia comentários sobre os ausentes ou atrasados: “Essa é a aluna $\mathrm{R}$, sumida, não veio a nenhum encontro no ano passado" ou "S sempre atrasada", ou ainda "G já virou 'veterano', não veio esse ano ainda".

Estudos sobre programas de mentoria em Medicina mostram que a insatisfação com a adesão dos alunos não foi um "privilégio" dessa mentora em especial. Malik ${ }^{18}$ relata que alguns alunos reconhecem a necessidade do mentoria, mas não consideram relevante participar, mesmo quando em dificuldades. Mentores entrevistados por Doubie et al. ${ }^{30}$ também referem enfrentar dificuldade com a adesão. De acordo com os mentores, as necessidades dos alunos são variadas, eles são muito ocupados e nem todos desejam esse tipo de relação.

Os alunos justificavam os atrasos e as faltas atribuindoos à rotina e à sobrecarga das atividades do curso e, no caso dos internos, à dificuldade em serem liberados dos estágios no hospital.

A aluna S, do terceiro ano, comenta: "Haja jogo de cintura! Estou com falta de tempo para tudo! Para a mentoria, estudar, dormir, nem vejo mais meus amigos". E continua: "A iniciação científica com a professora então, abandonei!". A mentora comenta: "Hoje tivemos oY, do quinto ano, que depois teve que ir embora: vida de quinto ano!" (OBS 4).

A falta de tempo, aqui destacada, é elemento central nos estudos sobre a qualidade de vida do estudante de Medicina ${ }^{31,32}$, dificultando que ele consiga cuidar da própria saúde, relacionar-se com a família e amigos, e desenvolver outros interesses. Em nosso estudo, as observações mostraram que a atividade de mentoria era também "contaminada" por esse contexto.

\section{A reunião não acaba quando termina}

A dinâmica do grupo, ao longo de reunião, seguia um curso de começo, meio e fim. O encontro iniciava-se, geralmente, "morno", "aquecia-se”, atingia um "ápice" e, ao final, apesar do cansaço dos membros, ainda se mostrava animado. Os alunos geralmente saíam juntos, conversando, e, algumas vezes, ficavam além do tempo, para conversar reservadamente com a mentora.

Já terminou o horário do encontro e todos estão motivados ouvindo X falar. Mas a mentora diz: "Bem, queridos, temos que terminar, pois tenho uma reunião agora". $X$ diz: "Vou voltar aqui para a gente conversar, preciso reclamar mais". Dra. A diz: "Isso, volte, e nós vemos o que pode ser feito". Os alunos saem da sala e continuam falando corredor afora (OBS 6).

\section{Como se relacionavam}

\section{Uma mentora muito motivada}

Após as queixas iniciais quanto às faltas e aos atrasos dos alunos, a mentora retomava o propósito do encontro: trocar ideias e experiências. 
Dra. A falou um pouco mais sobre a aluna ausente. Estava muito frustrada, mas conseguiu perguntar para a outra aluna: "E você o que tem feito?". A aluna C começa a falar animadamente, estava muito feliz: "Fui a uma visita ao hospital e adorei. Nossa! É muito grande.... A mentora a incentiva perguntando por onde ela passou, quando e como foi. Elas conversam animadamente. (OBS 1).

Era a mentora quem, na maioria das vezes, abria a discussão propondo um tema. Como os alunos chegavam de forma gradual, ela se preocupava sempre em informar aos atrasados o que havia sido discutido até então. Era muito importante para ela que o grupo, como um todo, compartilhasse o assunto em questão. Ela incentivava fortemente a troca de opiniões, ampliava as questões e fazia com que todos se expressassem a respeito. Havia também espaço para os temas levantados pelos alunos, assim como para que o grupo, sozinho, conduzisse as discussões. $O$ desentendimento e a crítica podiam aparecer, e discussões acaloradas aconteciam muitas vezes.

A mentora mostrava gostar de trabalhar em grupo, era empática, funcionava como um modelo de identificação, tinha senso de humor, capacidade de comunicação e habilidade para integrar e sintetizar. Contudo, algumas características de personalidade dos alunos, como a timidez e o retraimento, e certas atitudes ou comportamentos não eram bem-vindos. Por vezes, as dificuldades dela em lidar com esses aspectos geravam retraimento do grupo, nem sempre percebido por ela.

Essas observações ressaltam a importância de um espaço em que mentores possam refletir sobre suas intervenções. Freeman ${ }^{33}$, em sua experiência com mentores em programas de residência médica, destaca que o treinamento inicial é apenas uma fase de indução. $O$ suporte continuado, diz a autora, é fundamental, pois somente, à medida que ocorrem os encontros, os mentores conseguem identificar áreas ou atitudes que eles necessitam examinar, saber mais ou compreender melhor.

\section{Calouros e veteranos}

A relação calouros-veteranos era suportiva e marcada pela admiração e valorização. Os alunos dos primeiros anos escutavam muito interessados os mais velhos que respondiam a várias de suas dúvidas sobre o curso e "o que é necessário para ser médico". Os veteranos antecipavam não apenas os problemas, mas também os prazeres do futuro, estimulando os mais jovens a continuar em frente.

$Z$, sextoanista, diz: "O quarto ano foi o que mais gostei! Foi onde tudo começou a fazer sentido para mim, tudo o que eu havia aprendido nos outros anos! Por isso, não desistam, pois no começo o curso parece ruim, mas depois melhora!". Y, do quinto ano, concorda, e os calouros o escutam atentamente:
“Você passa por uma adaptação, no primeiro momento é tudo novidade, tudo está começando, nesta fase você se sente solto, às vezes até meio perdido, depois começa a aprender a propedêutica e aí já começa uma transição; no terceiro e quarto ano o curso dá uma virada, há mais contato com o que é ser médico, as coisas começam a fazer sentido, um maior contato com a prática e você se sente mais preparado. E depois vem o internato que junta vários ensinamentos do curso todo" (OBS 4).

A mentoria por pares $^{34}$, isto é, por meio da troca de experiência entrealunos de diferentes níveis acadêmicos, tal como ocorria informalmente nesse grupo, tem sido considerada uma estratégia bastante valiosa. Além dos benefícios para os alunos iniciantes, proporciona aos alunos veteranos o desenvolvimento de habilidades de colaboração, de responsabilidade e de comprometimento com o desenvolvimento de outra pessoa. Sprengel et al. ${ }^{35}$ consideram que a experiência favorece também atitudes positivas em relação à própria mentoria. Os alunos participantes podem, segundo esses autores, estar mais dispostos a procurar mentores ou a servir como mentores para os que entram na profissão.

\section{Sobre o que conversavam}

\section{De tudo um pouco}

O curso de Medicina e a rotina da faculdade eram temas constantes, ocupando a maior parte dos encontros. Conversavam muito sobre as disciplinas que estavam cursando, obrigatórias e optativas, a utilidade delas, a maneira como eram ministradas. Os alunos dos diferentes anos faziam comparações e discutiam mudanças, melhoras e pioras.

A mentora pergunta: "E na faculdade, as aulas? Como estão as coisas?". B, calouro, diz:"Neste semestre estou gostando bem mais!". Ele se anima e a outra caloura também. Dra. A pergunta:"O que mudou?". Ele responde:"Tudo! O esquema das aulas! Temos menos Bioquímica e mais casos clínicos!". D diz: "A prática ajuda a gente a querer estudar mais!". E complementa: "Dá ânimo! Agora você começa a estabelecer relações do que lê com o que vê na aula" (OBS 5).

As várias atividades extracurriculares a que os alunos se dedicavam, como ligas acadêmicas, iniciação científica, entre outras, eram foco de várias discussões. Os mais jovens, mesmo dentro de um curso já bastante exigente, mostravam forte interesse de se envolver em outras atividades, desejando complementar o curso, se integrar com outros alunos e, especialmente, se aproximar da prática médica.

$B$ pergunta sobre as ligas para seus colegas veteranos de mentoria. Z, do sexto ano, responde dizendo: "É bom você fazer, aproveitar os primeiros anos que são mais tranquilos. Veja bem, eu agora não tenho mais tempo e gostaria de ter feito mais. Faça, mesmo se for para você descobrir que não é aquilo que 
você quer! Sempre é muito válido!" (OBS 1).

O internato e a residência médica também eram assuntos que geravam muito interesse, curiosidade, entre os mais jovens, e preocupações, nos alunos dos últimos anos.

A mentora diz: "E os nossos alunos do quinto ano o que dizem sobre o internato?". X e Y começam falando da falta de tempo do quinto ano. Eles dizem que estão gostando e que agora se sentem médicos de verdade. Y diz: "Agora dá para sentir como é!". $Z$ fala: "Acho que é melhor aproveitar bem a faculdade, se enturmar com todos e aproveitar, pois agora, no quinto ano, sinto o estresse em relação à residência, você sente a competição aumentando, parece uma bomba-relógio". Todos escutam interessados. O encontro já passou do horário, e ninguém reclama. (OBS 2).

Assuntos de caráter pessoal também estavam presentes nas reuniões. A mentora se interessava pela família dos alunos, pelas férias deles, conversavam sobre esporte e lazer. A moradia dos alunos e as dificuldades de deslocamento até a faculdade também eram temas abordados, de modo a ajudar na adaptação dos calouros.

Dra. A diz: "R dê dicas para D sobre os melhores lugares para morar aqui por perto". R conta onde mora e relata que se locomove de metrô para ir à faculdade. $\mathrm{S}$ diz para ela: "Você inevitavelmente irá pegar trânsito, pois terá aulas na cidade universitária" (OBS 1).

A escolha da especialidade médica era tema fortemente presente nas reuniões. Os alunos mostravam interesse pela especialidade da mentora e pela atuação dela na área. Os mais novos desejavam saber sobre a opção de carreira futura dos internos, quando eles haviam se decidido e, especialmente, quais as áreas em que há maior qualidade de vida.

A mentora pergunta aos internos sobre a futura especialidade. $Z$ diz querer clínica médica, e X diz: "Eu não sei, na verdade estou quase desistindo da cirurgia". A mentora pergunta o porquê. $X$ responde: "Para ter uma melhor qualidade de vida". "E você, Y?" Ele responde: "Urologia, tenho um tio urologista, eu o admiro, ele consegue ter uma vida regrada, com hora para tudo" (OBS 9).

Os alunos dos últimos anos expressavam suas angústias em relação à residência médica discutindo sobre o que é necessário para passar no exame, o que deve haver no currículo, como se comportar na entrevista e, especialmente, se perguntando: "Será que eu fiz o suficiente?". Os alunos iniciantes acompanhavam essas questões com expressões de interesse, preocupação e solidariedade.

Z fala sobre os estudos para a prova de residência. B pergunta: "Quando foi que você se decidiu pela clínica médica?". Z responde: "Foi no quinto ano; antes eu achava que era ginecologia". B pergunta: "E quando você percebeu que estava virando médica?!" (OBS 6).

Tal diversidade das conversas nos encontros mostra a necessidade de estratégias que apoiem o desenvolvimento do aluno como um todo, não apenas o seu desempenho acadêmico ou suas habilidades clínicas. Nesse sentido, a mentoria apresenta-se como promotora de desenvolvimento, contrastando com outras abordagens de remediação de problemas. Não se trata de assistência para alunos considerados "em risco", nem de "fórum" para discussão e encaminhamento de problemas do curso. Como ressaltam Sandars et al. ${ }^{1}$, a escola médica articula a formação da identidade pessoal no início da vida adulta com toda uma variedade de experiências por ela apresentada. A mentoria, ao ser centrada na pessoa do aluno, ajuda os alunos a fazer sentido dos pensamentos, dos sentimentos e das ações derivados desse momento de crescimento pessoal e profissional.

\section{A relação com a observadora \\ Um feedback positivo}

O grupo focal para conversar sobre as observações realizadas e aprofundar a compreensão da relação de mentoria mostrou-se muito produtivo. A mentora e os alunos se reconheceram nos aspectos apresentados, os complementaram e, especialmente, os validaram.

O grupo comentou ter ficado surpreso com tudo o que foi observado. S. diz: "Você ficava aí sempre quietinha e, apesar do seu silêncio, observava tudo o que acontecia, em detalhes!". Comentaram a riqueza dos detalhes: "Tem coisas que nós fazemos e nem nos damos conta!", complementou $Y$.

\section{Quase um de nós}

Apesar do desafio que envolve observar e ser observado, o grupo assimilou a presença da observadora com naturalidade. O grupo agia espontaneamente em sua presença, e, com o tempo, parecia que a participação dela era esperada como se fosse membro do grupo.

$R$ disse: Fiquei com a impressão que a observadora frequentava diariamente o meu círculo de amizades: vocêfala dos nossos assuntos com tanta compreensão! Parece que você estava dentro das nossas conversas, nas salas de aula e pela faculdade! Também achei bem interessante como você consegue perceber as nossas angústias com tanta clareza.

Minayo et al. ${ }^{26}$, ao discutirem as fontes de informação na abordagem qualitativa, referem que, na observação de campo, "se focalizam comportamentos, relações e os imponderáveis da vida social", enquanto, no grupo focal, a técnica "se fundamenta na capacidade humana de formar opiniões e atitudes em interação" (p. 144). 
O feedback positivo dado à pesquisadora, além de reconhecer sua habilidade como observadora, mostra também, especialmente, a possibilidade de técnicas diferentes complementarem-se uma a outra, ampliando a compreensão do fenômeno e validando seus resultados.

\section{CONCLUSÕES}

Neste estudo de caso, buscamos, por meio de uma abordagem etnográfica complementada e validada por um grupo focal, aprofundar a compreensão das relações de mentoria em uma escola médica. Essa metodologia permitiu descrições detalhadas das interações, capturando aspectos que poderiam passar despercebidos se tratados apenas por meio de números ou avaliações a posteriori.

A expressão "olhar pelo buraco da fechadura" buscou aqui expressar a curiosidade do pesquisador e representar a aproximação da intimidade de algo que, no cotidiano dos programas de mentoria, acaba por ficar "escondido" - o que acontece em um encontro de mentoria enquanto ele naturalmente acontece.

O que mostrou o "buraco da fechadura"? O que nosso olhar conseguiu apreender?

A proximidade e a intimidade com o grupo de mentoria revelaram que grupos de mentoria, especialmente quando compostos por alunos de diferentes anos, são encontros de complexo manejo. Para dar conta desse desafio e desempenhar seu papel, o mentor deve acreditar no potencial da atividade de mentoria e, especialmente, ser capaz de fazer conexões entre os tópicos e as experiências de diferentes membros do grupo.

A partilha intergeracional de experiências entre os veteranos e aqueles dos anos iniciais permite que os alunos conheçam melhor o curso e discutam a formação médica a partir de diferentes perspectivas. Essa vivência compartilhada reduz a angústia dos alunos, permitindo que se identifiquem uns com os outros e percebam que não são os únicos com dificuldades, medos e inseguranças.

Sem dúvida, é necessário tempo, e, especialmente, é imprescindível que haja disponibilidade interna e externa para o desenvolvimento desse relacionamento. A estrutura e a dinâmica do curso médico têm grande impacto nos encontros e, ao mesmo tempo que geram a necessidade de mentoria, dificultam que os alunos usufruam plenamente de seus benefícios. Podemos dizer que a formação médica, ao mesmo tempo que "pede" a mentoria, a "impede", de certa forma.

É também necessário destacar que o espaço para a mentoria, mais do que físico, deve ser o de oferecer lugar e acolhimento para a real interlocução de diferentes ideias. A mentoria somente fará sentido e gerará interesse suficiente para ultrapassar as dificuldades de participação se os encontros possibilitarem a livre discussão de questões realmente interessantes, o prazer com o compartilhamento e reconhecimento de experiências comuns e, assim, a "descompressão" das angústias derivadas da formação.

\section{CONSIDERAÇÕES FINAIS}

Sem dúvida, futuros estudos são necessários para explorar a dinâmica de outros grupos de mentoria no mesmo programa e em outras escolas médicas.

A abordagem etnográfica, por meio de observação participante, é de custosa e difícil realização, mas adotá-la na compreensão das relações de mentoria pode enriquecer as investigações em profundidade do tema. Embora seja uma clássica metodologia de pesquisa, sua utilização neste estudo representa, de certa forma, uma "inovação" ao trazer, literalmente, um novo e raro "ponto de vista".

Acreditamos assim que, mesmo sendo derivada de um único estudo de caso, nossa investigação permite trazer algumas considerações práticas importantes para mentores, alunos e para a atividade de mentoria, na escola médica e em geral.

Ser um mentor efetivo e afetivo implica compreender o propósito da atividade, permanecer motivado, apresentar certas características pessoais, como generosidade, empatia, disponibilidade, flexibilidade e humildade, e, ao mesmo tempo, receber suporte ao longo do trabalho. Seria interessante se candidatos a mentor pudessem vivenciar, também como observadores participantes, alguns encontros de grupos de mentoria em funcionamento. Eles poderiam assim se aproximar da natureza da atividade grupal, se familiarizar com a proposta, suas potencialidades e limitações, fazendo assim uma "escolha informada".

Em relação aos alunos, vale a pena considerar a introdução de incentivos apropriados para que o contexto da formação médica não impeça aqueles potencialmente interessados, e com condições de aproveitar a mentoria, de participar da atividade. A participação em atividades de mentoria poderia ser formalmente reconhecida em momentos de avaliação do currículo ou do portfólio dos estudantes, reconhecendo que esses se preocuparam, ao longo do curso, com sua formação integral - cognitiva, técnica e humana.

Por fim, acreditamos que apoiar os alunos requer o desenvolvimento de uma cultura institucional em que tal postura seja considerada uma responsabilidade de todos. Para além do "buraco da fechadura" dos encontros de mentoria, o acolhimento e o desenvolvimento do futuro médico não deveriam ser tarefas exclusivas de mentores, mas sim uma disposição de todos os envolvidos nessa jornada: mentores, docentes, discentes e dirigentes. 


\section{AGRADECIMENTOS}

Agradecemos a participação, corajosa e autêntica, da mentora do grupo e de seus interessados e interessantes alunos.

\section{CONTRIBUIÇÃO DAS AUTORAS}

As duas autoras contribuíram para a concepção e o desenho do estudo. Fabiana Verardino Spina atuou como pesquisadora principal no projeto de pesquisa e realizou a coleta de dados. Fabiana Verardino Spina e Patrícia Lacerda Bellodi analisaram e interpretaram os dados. Fabiana Verardino Spina escreveu o primeiro rascunho do artigo, e Patrícia Lacerda Bellodi o revisou criticamente quanto à relevância do conteúdo. Ambas as autoras aprovaram a versão final do manuscrito.

\section{CONFLITO DE INTERESSES}

Declaramos não haver conflito de interesses.

\section{FINANCIAMENTO}

Conselho Nacional de Desenvolvimento Científico e Tecnológico (CNPq).

\section{REFERÊNCIAS}

1. Sandars J, Patel R, Steele H, McAreavey M. Association for Medical Education Europe. Developmental student support in undergraduate medical education: AMEE Guide No. 92. Med Teach. 2014;36(12):1015-26.

2. Frei E, Stamm M, Buddeberg-Fischer B. Mentoring programs for medical students - a review of the PubMed literature 2000-2008. BMC Med Educ. 2010;30;10:32.

3. Buddeberg-Fischer B, Herta KD. Formal mentoring programmes for medical students and doctors: a review of the Medline literature. Med Teach. 2006;28(3):248-57.

4. Taherian K, Shekarchian M. Mentoring for doctors. Do its benefits outweigh its disadvantages? Med Teach. 2008;30(4):e95-9.

5. Ehrich LC, Hansford B, Tennent L. Formal mentoring programs in education and other professions: a review of the literature. Educ Adm Q. 2004;40(4):518-40.

6. Lee A, Dennis C, Campbell P. Nature's guide for mentors. Nature 2007;447(7146):791-7.

7. Rhodes J. Stand by me: the risks and rewards of mentoring today's youth Cambridge: Harvard University Press; 2002.

8. Allen TD, Eby LT, O'Brien KE, Lentz E. The state of mentoring research: a qualitative review of current research methods and future research implications. J Vocat Behav. 2008;73(3):343-57.

9. Davis OC, Nakamura J. A proposed model for an optimal mentoring environment for medical residents: a literature review. Acad Med. 2010;85(6):1060-6.

10. Kalet A, Krackov S, Rey M. Mentoring for a new era. Acad Med. 2002;77(11):1171-2.

11. Murr AH, Miller C, Papadakis M. Mentorship through advisory colleges. Acad Med. 2002;77(11):1172-3.

12. Buddeberg-Fischer B, Vetsch E, Mattanza G. Career support in medicine: experiences with a mentoring program for junior physicians at a university hospital. Pyschosoc Med. 2004;1:Doc04.
13. von der Borch P, Dimitriadis K, Störmann S, Meinel FG, Moder S, Reincke M, et al. A novel large-scale mentoring program for medical students based on a quantitative and qualitative needs analysis. GMS Z Med Ausbild 2011;28(2):Doc26.

14. Kurré J, Bullinger M, Petersen-Ewert C, Guse AH. Differential mentorship for medical students: development, implementation and initial evaluation. Int J Med Educ. 2012;3:216-24.

15. Stenfors-Hayes T, Kalen S, Hult H, Dahlgren LO, Hindbeck H, Ponzer S. Being a mentor for undergraduate medical students enhances personal and professional development. Med Teach. 2010;32(2):148-53.

16. Meinel FG, Dimitriadis K, von der Borch P, Störmann S, Niedermaier S, Fischer MR. More mentoring needed? A cross-sectional study of mentoring programs for medical students in Germany. BMC Med Educ. 2011;11:68.

17. Straus SE, Johnson MO, Marquez C, Feldman MD. Characteristics of successful and failed mentoring relationships: a qualitative study across two academic health centers. Acad Med. 2013;88(1):82-9.

18. Malik S. Students, tutor, and relationships: the ingredients of a successful student support scheme. Med Educ. 2000;34(8):635-41.

19. Rabatin JS, Lipkin Jr M, Rubin AS, Schachter A, Nathan M, Kalet A. A year of mentoring in academic medicine: case report and qualitative analysis of fifteen hours of meetings between a junior and senior faculty member. J Gen Intern Med. 2004;19(5 Pt 2):569-73.

20. Hauer KE, Teherani A, Dechet A, Aagaard EM. Medical students'perceptions of mentoring: a focus-group analysis. Med Teach. 2005;27(8):732-4.

21. Steel T, Lämsä A, Jyrkinen M. Paradoxes of mentoring: an ethnographic study of a mentoring programme for highly-educated women with migrant backgrounds. Cult Unbound. 2019;11(2):275-97.

22. Snoeren MMWC, Raaijmakers R, Niessen TJH, Abma TA. Mentoring with(in) care: a co-constructed auto-ethnography of mutual learning. J Organ Behav. 2016;37(1):3-22.

23. Bellodi $\mathrm{PL}$, Martins MA, organizadores. Tutoria: mentoring na formação médica. São Paulo: Casa do Psicólogo; 2005.

24. Atkinson P, Pugsley L. Making sense of ethnography and medical education. Med Educ. 2005; 39(2):228-34.

25. Yin RK. Case study research: design and methods. London: Sage; 2001.

26. Minayo MCS, Costa AP. Fundamentos teóricos das técnicas de investigação qualitativa. Revista Lusófona de Educação. 2018;40(40):139-53.

27. Minayo MCS, organizadora. Pesquisa social: teoria, método e criatividade Petrópolis: Vozes; 2001.

28. Garnica AVM. Algumas notas sobre pesquisa qualitativa e fenomenologia Interface. 1997; 1(1):109-22.

29. Bardin L. Análise de conteúdo. Lisboa: Edições 70; 2008.

30. Doubie S, Smith S, Robins L. How assigned faculty mentors view their mentoring relationships: an interview study of mentors in medical education. Mentor Tutoring. 2010;18(4):337-59.

31. Tempski P, Bellodi PL, Paro HB, Enns SC, Martins MA, Schraiber LB. What do medical students think about their quality of life? A qualitative study. BMC Med Educ. 2012;12:106

32. Paro HB, Morales NM, Silva $\mathrm{CH}$, Rezende $\mathrm{CH}$, Pinto RM, Morales RR et al. Health-related quality of life of medical students. Med Educ. 2010;44(3):227-35.

33. Freeman R. Mentoring in general practice. Oxford: ButteworthHeinemann; 1998.

34. Taylor JS, Faghri S, Aggarwal N, Zeller K, Dollase R, Reis SP. Developing a peer-mentor program for medical students. Teach Learn Med 2013;25(1):97-102.

35. Sprengel AD, Job L. Reducing student anxiety by using clinical peer mentoring with beginning nursing students. Nurse Educ. 2004;29(6):246-50. 